\title{
Comparison of the efficacy and safety of the EC-T (epirubicin/ cyclophosphamide followed by docetaxel) and TCb (docetaxel/carboplatin) neoadjuvant regimens in early TOP2A-normal stage II-III breast cancer
}

\author{
W. P. $\mathrm{LI}^{1,2, *}$, T. ZHU ${ }^{1, *}$, M. X. HU ${ }^{1}$, M. YANG ${ }^{1}$, F. JI ${ }^{1}$, H. F. GAO ${ }^{1}$, C. Q. YANG ${ }^{1}$, L. L. ZHANG ${ }^{1}$, M. Y. CHENG ${ }^{1}$, F. P. XU ${ }^{3, *}$, K. WANG $^{1,2, *}$ \\ ${ }^{1}$ Department of Breast Cancer, Cancer Center, Guangdong Provincial People’s Hospital, Guangdong Academy of Medical Sciences, Guangzhou, \\ China; ${ }^{2}$ The Second School of Clinical Medicine, Southern Medical University, Guangzhou, China; ${ }^{3}$ Department of Pathology, Cancer Center, \\ Guangdong Provincial People's Hospital, Guangdong Academy of Medical Sciences, Guangzhou, China
}

${ }^{*}$ Correspondence: gzwangkun@126.com,xfpraider@tom.com

"Contributed equally to this work.

Received January 30, 2020/ Accepted April 8, 2020

\begin{abstract}
This study aimed to compare the efficacy and safety of the EC-T (4 cycles of epirubicin $90 \mathrm{mg} / \mathrm{m}^{2}+$ cyclophosphamide $600 \mathrm{mg} / \mathrm{m}^{2}$, followed by 4 cycles of docetaxel $\left.75 \mathrm{mg} / \mathrm{m}^{2}\right)$ and TCb $\left(6\right.$ cycles of docetaxel $75 \mathrm{mg} / \mathrm{m}^{2}$, intravenous drip (ID), day $1+$ carboplatin AUC 6, ID, day 1) neoadjuvant regimens in patients with TOP2A-normal stage II-III breast cancer. This study analyzed 280 patients enrolled from three studies registered with ClinicalTrials.gov (NCT03140553, NCT03154749, NCT03507465) with early TOP2A-normal stage II-III breast cancer who received neoadjuvant chemotherapy, including 100 patients who received the EC-T regimen and 180 patients who received the TCb regimen. The primary endpoint was the ratio of RCB 0/1 (residual cancer burden 0/1) after neoadjuvant chemotherapy. The secondary endpoint was the safety of the two groups. There was no significant difference in the ratio of RCB $0 / 1$ between the two groups ( $23 \%$ vs. $23.9 \%, p=0.614$ ). Among the triple-negative breast cancer patients, the efficacy did not differ between the two groups ( $40 \%$ vs. $32 \%, p=0.52$ ). Among the lymph node metastasis patients, the efficacy of the EC-T group was significantly better than that of the TCb group ( $14 \%$ vs. $2.6 \%, \mathrm{p}=0.03$ ). Regarding the side effects, the incidence of grade $3 / 4$ anemia was higher in the EC-T group than in the TCb group $(21.0 \%$ vs. $8.33 \%, p=0.002)$, while the incidence of grade $3 / 4$ neutropenia was higher in the EC-T group than in the TCb group ( $17 \%$ vs. $14.44 \%, \mathrm{p}=0.570$ ), and the incidence of grade $3 / 4$ thrombocytopenia was low in each group (EC-T group: $6 \%$ and TCb group: $7.22 \%, \mathrm{p}=0.697$ ). In the EC-T group, grade $3 / 4$ nausea and vomiting occurred in 5 patients. The EC-T group showed a higher rate of grade $3 / 4$ myalgia than the TCb group ( $7 \%$ and $4.44 \%$, respectively, $\mathrm{p}=0.363$ ). To conclude, the TCb regimen can be used as an alternative regimen for TOP2A-normal stage II-III breast cancer patients in neoadjuvant chemotherapy. However, in patients with node-positive tumors, EC-T is still recommended. Though no difference of grade $3 / 4$ thrombocytopenia in two groups, grade 4 thrombocytopenia caused by the carboplatincontaining regimen should be taken seriously.
\end{abstract}

Key words: TOP2A-normal, epirubicin, docetaxel, neoadjuvant, residual cancer burden

Anthracyclines were first used in breast cancer in the 1950s. Although they may cause major blood-related toxicity and cardiotoxicity, they are currently the most widely used chemotherapy drugs in breast cancer patients [1]. Metaanalyses on data obtained from 123 studies have shown that early breast cancer patients can benefit from docetaxel or anthracyclines [2], and the sensibility to anthracyclines varies according to molecular type in breast cancer patients. Therefore, it is possible to avoid unnecessary treatment if patients who are insensitive to anthracyclines are screened out by molecular markers.
The gene that encodes TOP $2 \mathrm{~A}$ is located at $17 \mathrm{q} 12 \mathrm{q}-21$ and plays a key role in cell division [3]. TOP2A gene amplification often predicts a worse prognosis and stronger tumor aggressiveness in breast cancer patients [4]. A large number of clinical trials have shown that although abnormal expression of the TOP2A gene may imply a poor prognosis, it can also improve the efficacy of anthracyclines $[5,6]$, suggesting that more patients with a normal TOP2A gene may be exempted from anthracycline chemotherapy. Previous studies have shown that in breast cancer patients, the proportion of TOP2A-normal patients is nearly $80 \%[7,8]$, and the 
percentage of TOP2A gene amplification is only $12-24 \%$ [5, $8,9]$. There is currently a lack of relative data on whether breast cancer patients with normal expression of TOP2A can benefit from anthracyclines.

Neoadjuvant chemotherapy can predict the efficacy of drugs in patients with breast cancer earlier; it is increasingly used in patients with early breast cancer. RCB can predict the prognosis of each molecular subtype by assessing residual tumors in the breast and axilla after neoadjuvant chemotherapy [10]. It can more accurately predict the prognosis of patients who have not achieved pCR after neoadjuvant chemotherapy, which is a great supplement to the current evaluation system after neoadjuvant chemotherapy. Therefore, this study aims to compare the efficacy of neoadjuvant chemotherapy with anthracyclines and non-anthracyclines in patients with TOP2A-normal breast cancer from three prospectively clinical trials (NCT03140553, NCT03154749, NCT03507465). NCT03140553 was a randomized trial of TCH (Docetaxel/Carboplatin/Trastuzumab) Versus EC-TH (Epirubicin/Cyclophosphamide followed by Docetaxel/ Trastuzumab) as Neoadjuvant Treatment for HER2-Positive Breast Cancer. NCT03154749 was a phase II prospective randomized trial of docetaxel in combination with carboplatin $(\mathrm{TCb})$ versus EC followed by docetaxel as neoadjuvant chemotherapy for triple-negative, early-stage breast cancer. NCT03507465 was a randomized trial of Letrozole Plus Low-Dose Metronomic Capecitabine versus EC-T (Epirubicin/Cyclophosphamide Followed by Docetaxel) as Neoadjuvant Therapy for ER+/HER2-negative Breast Cancer. We enrolled the participants with normal TOP-2A from these clinical trials.

\section{Patients and methods}

Patients. Based on the data from three studies registered with ClinicalTrials.gov (NCT03140553, NCT03154749, NCT03507465), a total of 280 breast cancer patients with normal TOP2A expression and who received neoadjuvant chemotherapy at Guangdong Provincial People's Hospital were enrolled and included in the study. Inclusion criteria were as follows: 1) Thick needle-proven primary carcinoma (breast cancer) with normal expression of TOP2A; 2) Imaging confirmed as stage II-III breast cancer; 3) Age 18-70 years old; 4) No distant metastasis; and 5) Good bone marrow reserve and heart functions. Exclusion criteria were as follows: 1) Sentinel lymph node biopsy before chemotherapy; 2) Other locations of tumors in the past 5 years; and 3) Relevant antitumor treatment.

Chemotherapy regimen. A total of 180 patients received the $\mathrm{TCb}$ regimen $\left(75 \mathrm{mg} / \mathrm{m}^{2}\right.$ docetaxel, ID, day $1+$ carboplatin AUC6, ID, day 1), and 100 patients received EC-T (4 cycles of epirubicin $90 \mathrm{mg} / \mathrm{m}^{2}+$ cyclophosphamide $600 \mathrm{mg} / \mathrm{m}^{2}$, followed by 4 cycles of docetaxel $75 \mathrm{mg} / \mathrm{m}^{2}$ ). Seventy-five HER2 + patients were also treated with Herceptin $\left(8 \mathrm{mg} / \mathrm{kg}\right.$ Herceptin for the first time, $6 \mathrm{mg} / \mathrm{m}^{2}$ maintenance dose on day 2). All patients received $300 \mu \mathrm{g} /$ day of G-CSF prophylactic treatment on days 2 and 3 after chemotherapy. The dose of chemotherapy was adjusted according to the inspection results on the first day of each scheduled chemotherapy. When neutrophils, hemoglobin, platelets, and other non-blood-related adverse reactions showed the toxicity of 3 or more degrees (according to the guidelines of RECIST 1.1), chemotherapy was delayed by one week. B-ultrasound was made for staging evaluation before and after neoadjuvant chemotherapy.

Clinical pathological assessment. The kits used for TOP2A gene detection (TOP2A FISH pharmDx) and HER2 gene detection (HER2 FISH pharmDx) were obtained from DAKO, and fluorescence in situ hybridization was performed strictly in accordance with the operating instructions. Under the microscope, the orange fluorescence represents the TOP2A or HER2 gene probe signal, and green fluorescence represents the chromosome 17 probe signal. The TOP2A state was determined based on the ratio of the mean number of TOP2A gene probe signals per cell to the mean number of chromosome 17 probe signals. When the ratio of the TOP2A gene probe signal to the chromosome 17 probe signal is between 0.8 and 2.0 , the TOP2A gene is in a normal state. By the IHC method, the critical value of ER (estrogen receptor) and $\mathrm{PgR}$ (progesterone receptor) interpretation is $10 \%$ tumor cell staining; HER2 (human epidermal growth factor receptor 2)-positive is defined as $\mathrm{IHC}+++$ or $\mathrm{IHC}++$, FISH-positive; HER2-negative is defined as IHC $0-+$ or IHC++, FISH-negative; and HER2 critical is IHC++, with FISH representing the critical value [11]. High expression of Ki67 is defined as $\geq 15 \%$, and low expression of Ki67 is defined as $<15 \%$. According to the results of immunohistochemistry, breast cancer is divided into five types: Luminal A, Luminal B (HER2-), Luminal B (HER2+), HER2+, and triple-negative. Molecular subtype typing standards are as follows: Luminal A: ER- and/or PR-positive, HER2-negative, Ki67 <15\%; Luminal B (HER2-): ER- and/or PR-positive, HER2-negative, Ki67 $\geq 15 \%$; Luminal B (HER2+): ER- and/ or PR-positive, HER2-positive, Ki67 $\geq 15 \%$; HER2+: ER- and PR-negative, HER2-positive; and triple-negative: ER-, PRand HER2-negative.

Statistical analysis. The primary endpoint of this study was the RCB 0/1. Although pCR and RCB appear to be suboptimal prognostic indicators for breast cancer, the extent of RCB is a significant prognostic biomarker [10]. The RCB scoring system is mainly based on the largest diameter of the residual tumor bed, the cellularity of the tumor bed, and the number and size of metastatic lymph nodes after neoadjuvant chemotherapy, among which pCR is judged as RCB 0 . According to the scoring system, the cutoff point of RCB I and RCB II is 1.36, the cut-off point of RCB II and RCB III is 3.28 [12]. The patient's RCB index is obtained after two doctors interpret pathological sections without intervention, which are reviewed by a pathologist. We used the chi-square test to analyze the choice of chemotherapy regimen and clinical 
features and the association between molecular markers and the acquisition of RCB 0/1. Two-sided $p<0.05$ in the statistical test results was considered statistically significant.

\section{Results}

We totally analyzed 369 TOP2A-normal breast cancer patients who received neoadjuvant therapy from 3 clinical trials (NCT03140553, NCT03154749, NCT03507465); excluding 52 patients with TOP2A amplification or TOP2A deletion, 29 patients who received neoadjuvant endocrine therapy, 8 patients who did not undergo surgery at our center. Finally, 280 patients with TOP2A-normal breast cancer who received neoadjuvant chemotherapy were analyzed. Among them, 100 received EC-T and 180 received TCb (Figure 1). The median age was 44 years, of which 172 (61.4\%) patients underwent a mastectomy and $108(38.6 \%)$ received breastconserving surgery. The clinicopathological features of the patients are shown in Table 1. A total of 75 HER2-positive patients were treated with trastuzumab.

Efficacy Response. The proportion of patients with RCB $0 / 1$ in the two groups was $23 \%$ (EC-T group) and $23.9 \%$ ( $\mathrm{TCb}$ group, $\mathrm{p}=0.614)$. There was no significant difference between the two groups.

Exploratory studies found no significant difference in efficacy between the two chemotherapy regimens for any of the breast cancer subgroups (including tumor size, tumor histological grade, hormone receptor status, Ki67 expression, and menopausal status), apart from nodal status (Figure 2). Among the triple-negative breast cancer patients, the efficacy of the TCb group was not superior to that of the EC-T group $(\mathrm{p}=0.52)$. In patients with lymph node metastasis, the efficacy of the EC-T group was significantly better than that of the

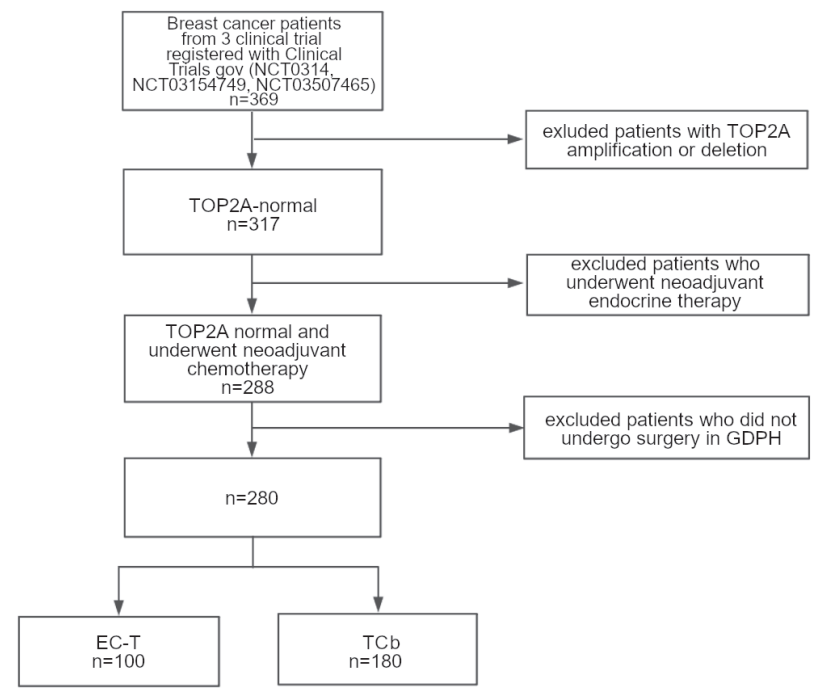

Figure 1. Study flowchart. GDPH - Guadong Provincial People's Hospital, EC-T - epirubicin/cyclophosphamide followed by docetaxel, TCb - docetaxel/carboplatin
$\mathrm{TCb}$ group $(\mathrm{p}=0.03)$. The recurrence-free survival analysis between the EC-T group and TCb group was examined by using the Kaplan-Meier method with the log-rank test. The median follow-up was 41.5 months. There was no significant difference between the two groups in recurrence-free survival (RFS, $\mathrm{p}=0.848$, Figure 3 ).

Table 1. Patient characteristics.

\begin{tabular}{|c|c|c|}
\hline Characteristic & Anthracycline & Non-anthracycline \\
\hline \multicolumn{3}{|l|}{ Menstrual status } \\
\hline Premenopause & $57(57 \%)$ & $108(60 \%)$ \\
\hline Postmenopause & $43(43 \%)$ & $72(40 \%)$ \\
\hline \multicolumn{3}{|l|}{ Grade } \\
\hline 1 & $4(4 \%)$ & $6(4 \%)$ \\
\hline 2 & $58(58 \%)$ & $87(48 \%)$ \\
\hline 3 & $38(38 \%)$ & $87(48 \%)$ \\
\hline \multicolumn{3}{|l|}{ Tumor size $(\mathrm{cm})$} \\
\hline$\leq 2$ & $6(6 \%)$ & $18(10 \%)$ \\
\hline$>2, \leq 5$ & $71(71 \%)$ & $148(82 \%)$ \\
\hline$>5$ & $23(23 \%)$ & $14(8 \%)$ \\
\hline \multicolumn{3}{|l|}{ Lymph node status } \\
\hline Positive & $50(50 \%)$ & $77(43 \%)$ \\
\hline Negative & $50(50 \%)$ & $103(57 \%)$ \\
\hline \multicolumn{3}{|l|}{ Stage } \\
\hline$\alpha$ & $87(87 \%)$ & $168(93 \%)$ \\
\hline$\beta$ & $13(13 \%)$ & $12(7 \%)$ \\
\hline \multicolumn{3}{|l|}{ Molecular subtyping } \\
\hline Luminal A & $10(10 \%)$ & $15(8 \%)$ \\
\hline Luminal B (Her2-) & $43(43 \%)$ & $68(38 \%)$ \\
\hline Luminal B (Her2+) & $13(13 \%)$ & $30(17 \%)$ \\
\hline Her2+ & $9(9 \%)$ & $22(12 \%)$ \\
\hline TNBC & $25(25 \%)$ & $45(25 \%)$ \\
\hline \multicolumn{3}{|l|}{ ER status } \\
\hline Negative & $31(31 \%)$ & $70(39 \%)$ \\
\hline Positive & $69(69 \%)$ & $110(61 \%)$ \\
\hline \multicolumn{3}{|l|}{ PgR status } \\
\hline Negative & $45(45 \%)$ & $82(46 \%)$ \\
\hline Positive & $55(55 \%)$ & $98(54 \%)$ \\
\hline \multicolumn{3}{|l|}{ HER2 status } \\
\hline Negative & $78(78 \%)$ & $127(71 \%)$ \\
\hline Positive & $22(22 \%)$ & $53(29 \%)$ \\
\hline \multicolumn{3}{|l|}{ Ki67 } \\
\hline High & $66(66 \%)$ & $126(70 \%)$ \\
\hline Low & $34(34 \%)$ & $54(30 \%)$ \\
\hline \multicolumn{3}{|l|}{ Surgical treatment } \\
\hline Mastectomy & $65(65 \%)$ & 107 (59\%) \\
\hline Lumpectomy & $35(35 \%)$ & $73(41 \%)$ \\
\hline \multicolumn{3}{|l|}{$\mathrm{RCB}$} \\
\hline $0 / 1$ & $23(23 \%)$ & $43(23.9 \%)$ \\
\hline 2 & $67(67 \%)$ & $121(67.2 \%)$ \\
\hline 3 & $10(10 \%)$ & $16(8.9 \%)$ \\
\hline
\end{tabular}

Abbreviations: ER - estrogen receptor, PgR - progesterone receptor, HER2 - human epidermal growth factor receptor 2 


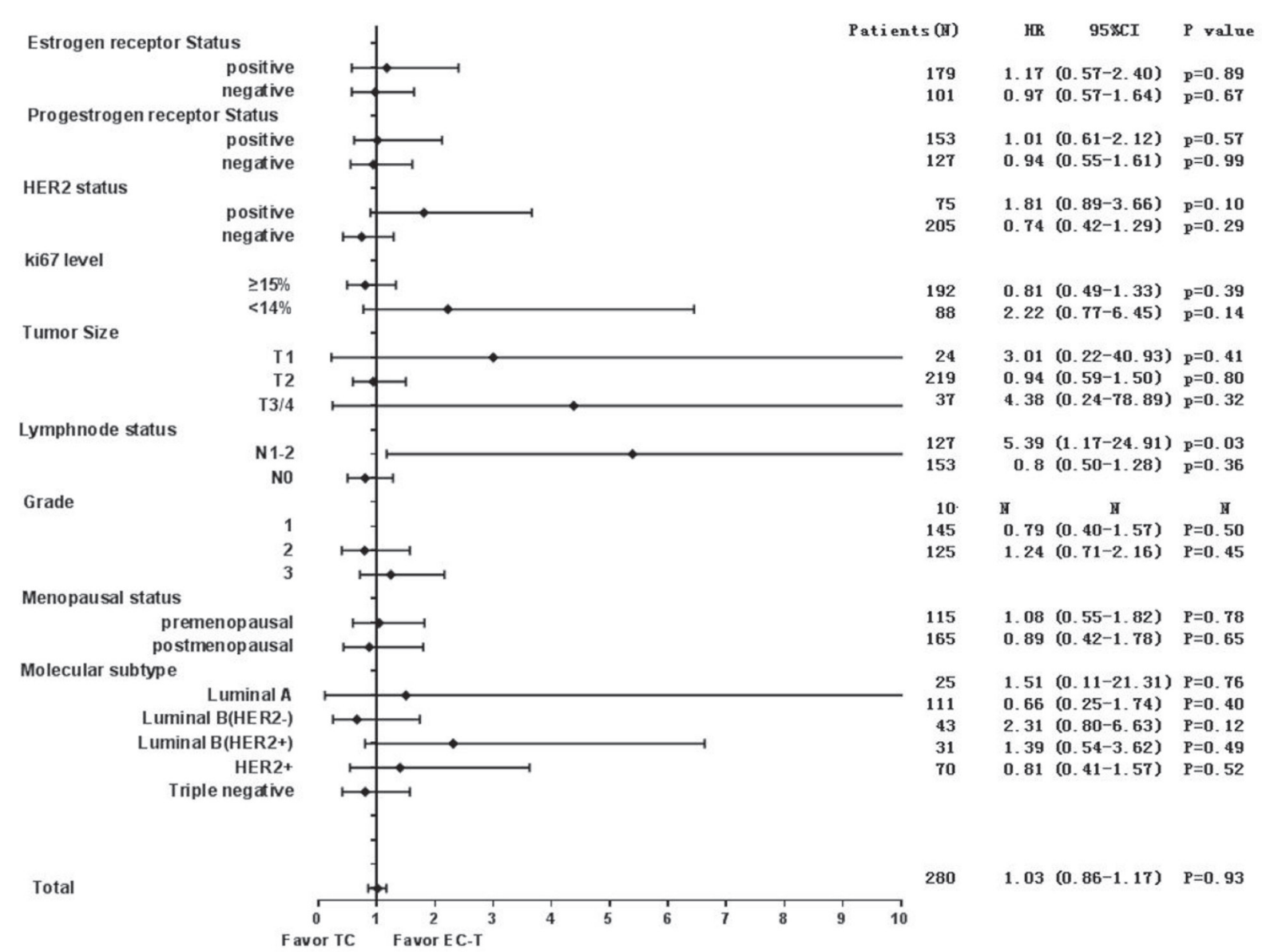

Figure 2. Forest plot shows proportional hazards models for RCB0/1 according to estrogen receptor status, progesterone receptor status, Ki67, HER2, tau, BCL-2 status, tumor size, lymph node status, malignancy grade, menopausal status, and molecular subtype. EC-T - epirubicin/cyclophosphamide followed by docetaxel, TC - docetaxel/carboplatin, HER2 - human epidermal growth factor receptor 2, tau - a microtubule-associated protein

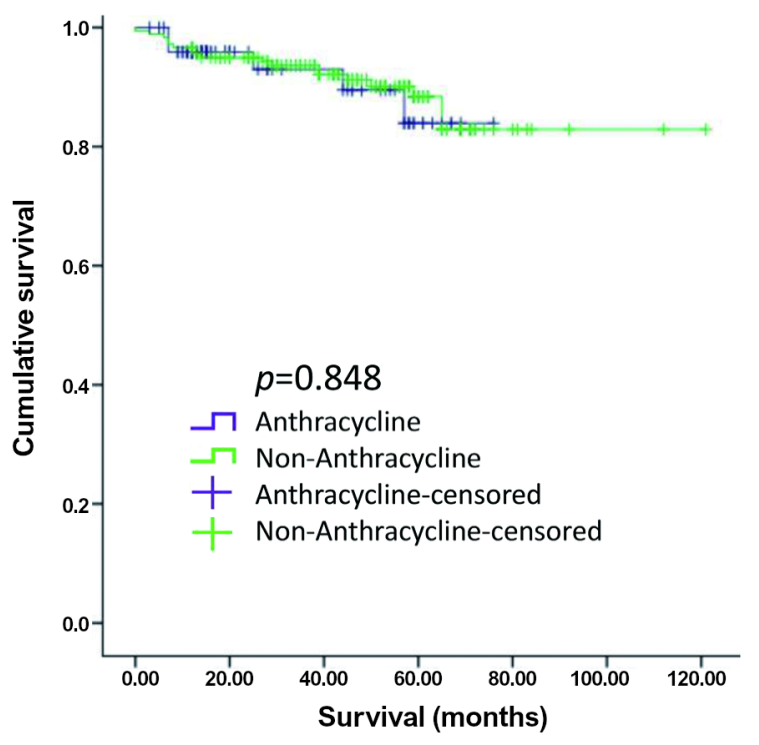

Figure 3. Comparison of RFS (recurrence-free survival) between Anthracycline- and Non-Anthracycline group.
Toxicity. In terms of hematologic toxicity, despite the routine use of G-CSF prophylactic treatment in both arms, grade 3-4 neutropenia still occurs, and our study shows that the incidence of grade 3-4 neutropenia was higher in the EC-T group than in the TCb group ( $17 \%$ and $14.44 \%$, respectively, $\mathrm{p}=0.570$ ). Regarding the side effects, the incidence of grade $3 / 4$ anemia was higher in the EC-T group than in the TCb group $(21.0 \%$ vs. $8.33 \%, p=0.002)$. The incidence of grade 3-4 thrombocytopenia was low in each group (EC-T: $6 \%$ and TCb: $7.22 \%$, respectively, $\mathrm{p}=0.697$ ). However, 2 patients suffered grade 4 thrombocytopenia and delayed chemotherapy in the TCb group. One patient in the $\mathrm{TCb}$ group experienced grade 3 liver damage. No severe cardiac damage was detected during chemotherapy in both groups.

As for nonhematologic toxicity, both groups of patients tolerated the chemotherapy regimens well. In the EC-T group, grade 3/4 nausea and vomiting occurred in 5 patients, no one showed grade $3 / 4$ nausea and vomiting in the $\mathrm{TCb}$ group. The EC-T group showed a higher rate of grade 3/4 myalgia than the $\mathrm{TCb}$ group ( $7 \%$ and $4.44 \%$, respectively, $\mathrm{p}=0.363$, Table 2). 


\section{Discussion}

In this study, 280 patients were finally analyzed from three clinical trials, of which 100 patients received the EC-T regimen with neoadjuvant chemotherapy and 180 patients received the $\mathrm{TCb}$ regimen with neoadjuvant chemotherapy. There was no significant difference in the ratio of RCB $0 / 1$ between the two groups (23\% for EC-T group vs. $23.9 \%$ for $\mathrm{TCb}$ group, $\mathrm{p}=0.614)$. This result confirmed that the anthracycline-based regimen is not the only option in the neoadjuvant chemotherapy treatment of patients with normal TOP2A.

Previous studies have shown that patients with triplenegative and HER2+ breast cancer who achieved pCR after neoadjuvant chemotherapy had a better prognosis, whereas in patients with luminal breast cancer, the predictive effect of pCR was significantly lower [13], with a pCR of only $12-16 \%$ [14]. Our previous report showed that luminal patients receiving the $\mathrm{TCb}$ regimen with neoadjuvant chemotherapy had a pCR of only 7-12\% [15]. There were good prognoses for HR+/HER2- patients who achieved PCR or RCB-I, among whom 5 years of RFS were $88 \%$ and $100 \%$, respectively [10]. For patients who cannot obtain pCR, RCB can better predict their prognosis and chemotherapy efficacy. In breast cancer patients excluding HR+/HER2-, the prognosis of RCB 0 was not better than that of RCB-I, and the prognosis of RCB 0/1 was significantly better than that of RCB 2/3 [10]. Therefore, this study used RCB $0 / 1$ as the primary endpoint after neoadjuvant chemotherapy and hoped to explore the association between RCB $0 / 1$ and prognosis at a later date. It is also important to note that using the RCB scoring system may increase the burden on the pathologist. In this study, the RCB score was carefully judged by three doctors, including a review by a pathologist.

Previous studies have shown that paclitaxel combined with carboplatin can achieve significant efficacy and good tolerance in breast cancer patients [16-18]. In this study, two groups of patients obtained a similar RCB 0/1 ratio. In the subgroup analysis, triple-negative breast cancer patients were more likely to benefit from the TCb regimen. GeparSixto trial has shown that in patients with the triplenegative type, using carboplatin in neoadjuvant chemotherapy can achieve a higher rate of complete pathologic response [17]. Among lymph node-positive patients, the EC-T regimen was significantly better than the $\mathrm{TCb}$ regimen $(p=0.03)$. Although a great deal of research has shown that the addition of carboplatin to standard neoadjuvant chemotherapy for TNBC significantly improves pCR rates, there is still no direct evidence that $\mathrm{TCb}$ neoadjuvant chemotherapy is superior to $\mathrm{EC}-\mathrm{T}$ in triple-negative patients. In this study, the efficacy of the $\mathrm{TCb}$ group was not superior to that of the EC-T group $(\mathrm{p}=0.52)$ among the triple-negative breast cancer patients, Moreover, it should be taken seriously when using a non-anthracycline regimen in node-positive patients.
Table 2. Toxicity.

\begin{tabular}{lccc}
\hline Grade 3/4 & Anthracycline & Non-Anthracycline & p-value \\
Advent event & $\mathbf{n}(\%)$ & $\mathbf{n}(\%)$ & 0.570 \\
\hline Neutropenia & $17(17.00)$ & $26(14.44)$ & $<0.001$ \\
Leucopenia & $13(13.00)$ & $4(2.22)$ & 0.002 \\
Anemia & $21(21.00)$ & $15(8.33)$ & 0.697 \\
Thrombocytopenia & $6(6.00)$ & $13(7.22)$ & 0.643 \\
ALT & - & $1(0.56)$ & 0.045 \\
Fever & $3(3.00)$ & - & 0.005 \\
Nausea/Vomiting & $5(5.00)$ & - & 0.357 \\
Constipation & $1(1.00)$ & - & 0.127 \\
Diarrhea & $2(2.00)$ & - & 0.363 \\
Myalgia & $7(7.00)$ & $8(4.44)$ & 0.045 \\
Bone pain & $3(3.00)$ & - & 0.619 \\
Fatigue & $2(2.00)$ & $2(1.11)$ & - \\
Dyspnea & - & - & - \\
Cough & - & - & 0.670 \\
Neuropathy & $-3.00)$ & - & - \\
Cardiac impairment & - & $-67)$ & - \\
\hline
\end{tabular}

In terms of hematologic toxicity, the anthracyclinecontaining regimen may have a higher rate of 3/4 neutropenia than the other group ( $17 \%$ vs. $14.44 \%, p=0.570)$. In the TCb group, 2 patients had severe thrombocytopenia and delayed chemotherapy. Although the incidence of grade 3/4 thrombocytopenia was low in each group, attention must be paid to the patients who experienced grade $3 / 4$ thrombocytopenia in the TCb group. Regarding nonhematologic toxicity, the incidence of grade 3-4 adverse events was low, while the subjective expression of discomfort (including grade $1 / 2$ abdominal pain, fatigue, hand and foot soreness, and muscle pain) was higher, which might be related to patient complaints (patient reported) [19]. The incidence of hand and foot soreness after sequential docetaxel was $3 \%$ in EC-T chemotherapy, while it was $1.67 \%$ in the $\mathrm{TCb}$ group, and most of the adverse reactions occurred after the end of the fifth chemotherapy cycle (the first time receiving docetaxel chemotherapy), which is similar to the results of the DBCG07-READ test [20]. The possible causes are as follows: 1. EC-T has a longer chemotherapy cycle than the $\mathrm{TCb}$ regimen; 2 . The dose of docetaxel in the EC-T regimen exceeds that of the $\mathrm{TCb}$ regimen. The $\mathrm{EC}-\mathrm{T}$ group showed a higher rate of grade $3 / 4$ myalgia than the TCb group ( $7 \%$ and $4.44 \%$, respectively) though the incidence of each group is low. To solve this problem, in addition to wearing ice gloves and elastic stockings [21], acupuncture treatment was used for peripheral nerve pain caused by taxanes in our study [22], and patients generally have significant relief of symptoms after one week of treatment.

This study was based on three prospectively clinical trials (NCT03140553, NCT03154749, NCT03507465). However, 
the imbalance between the two groups may also cause bias in results. In addition, there have been few studies that selected RCB $0 / 1$ as the endpoint, and more trial verification is needed. The study also required long-term survival followup to verify differences in efficacy between the two groups.

In summary, the $\mathrm{TCb}$ regimen may be an alternative neoadjuvant chemotherapy regimen for breast cancer patients with normal TOP2A. In this study, the TCb regimen achieved a similar ratio of RCB $0 / 1$ to the EC-T regimen, and the adverse effects were relatively mild. However, more attention is still required for the toxicity of thrombocytopenia caused by carboplatin.

Acknowledgments: The funding source had no role in this study. All the authors had final responsibility for the decision to submit this manuscript for publication. We thank all the patients analyzed in this study and all the physicians for their work.

\section{References}

[1] GIANNI L, NORTON L, WOLMARK N, SUTER TM, BONADONNA G et al. Role of anthracyclines in the treatment of early breast cancer. J Clin Oncol 2009; 27: 4798-4808. https://doi.org/10.1200/JCO.2008.21.4791

[2] EARLY BREAST CANCER TRIALISTS' COLLABORATIVE GROUP (EBCTCG), PETO R, DAVIES C, GODWIN J, GRAY R et al. Comparisons between different polychemotherapy regimens for early breast cancer: meta-analyses of long-term outcome among 100,000 women in 123 randomised trials. Lancet 2012; 379: 432-444. https://doi. org/10.1016/S0140-6736(11)61625-5

[3] Wang JC. Cellular roles of DNA topoisomerases: a molecular perspective. Nature reviews Nat Rev Mol Cell Biol 2002; 3: 430-440. https://doi.org/10.1038/nrm831

[4] SHVERO J, KOREN R, SHVILI I, YANIV E, SADOV R et al. Expression of human DNA Topoisomerase II-alpha in squamous cell carcinoma of the larynx and its correlation with clinicopathologic variables. Am J Clin Pathol 2008; 130: 934-939. https://doi.org/10.1309/AJCPROG61USKCBEI

[5] DURBECQ V, PAESMANS M, CARDOSO F, DESMEDT C, DI LEO A et al. Topoisomerase-II alpha expression as a predictive marker in a population of advanced breast cancer patients randomly treated either with single-agent doxorubicin or single-agent docetaxel. Mol Cancer Ther 2004; 3: 1207-1214.

[6] BRASE JC, SCHMIDT M, FISCHBACH T, SÜLTMANN $\mathrm{H}, \mathrm{BOJAR} \mathrm{H}$ et al. ERBB2 and TOP2A in breast cancer: a comprehensive analysis of gene amplification, RNA levels, and protein expression and their influence on prognosis and prediction. Clin Cancer Res 2010; 16: 2391-2401. https://doi. org/10.1158/1078-0432.CCR-09-2471

[7] FRITZ P, CABRERA CM, DIPPON J, GERTEIS A, SIMON $\mathrm{W}$ et al. c-erbB2 and topoisomerase IIalpha protein expression independently predict poor survival in primary human breast cancer: a retrospective study. Breast Cancer Res 2005; 7: R374-384. https://doi.org/10.1186/bcr1012
[8] KNOOP AS, KNUDSEN H, BALSLEV E, RASMUSSEN BB, OVERGAARD J et al. retrospective analysis of topoisomerase IIa amplifications and deletions as predictive markers in primary breast cancer patients randomly assigned to cyclophosphamide, methotrexate, and fluorouracil or cyclophosphamide, epirubicin, and fluorouracil: Danish Breast Cancer Cooperative Group. J Clin Oncol 2005; 23: 7483-7490. https://doi.org/10.1200/JCO.2005.11.007

[9] O'MALLEY FP, CHIA S, TU D, SHEPHERD LE, LEVINE $\mathrm{MN}$ et al. Topoisomerase II alpha and responsiveness of breast cancer to adjuvant chemotherapy. J Natl Cancer Inst 2009; 101: 644-650. https://doi.org/10.1093/jnci/djp067

[10] SYMMANS WF, WEI C, GOULD R, YU X, ZHANG Y et al. Long-Term Prognostic Risk After Neoadjuvant Chemotherapy Associated With Residual Cancer Burden and Breast Cancer Subtype. J Clin Oncol 2017; 35: 1049-1060. https:// doi.org/10.1200/JCO.2015.63.1010

[11] WOLFF AC, HAMMOND ME, HICKS DG, DOWSETT M, MCSHANE LM et al. Recommendations for human epidermal growth factor receptor 2 testing in breast cancer: American Society of Clinical Oncology/College of American Pathologists clinical practice guideline update. J Clin Oncol 2013; 31: 3997-4013. https://doi.org/10.1200/ JCO.2013.50.9984

[12] SYMMANS WF, PEINTINGER F, HATZIS C, RAJAN R, KUERER $\mathrm{H}$ et al. Measurement of residual breast cancer burden to predict survival after neoadjuvant chemotherapy. J Clin Oncol 2007; 25: 4414-4422. https://doi.org/10.1200/ JCO.2007.10.6823

[13] CORTAZAR P, ZHANG L, UNTCH M, MEHTA K, COSTANTINO JP et al. Pathological complete response and long-term clinical benefit in breast cancer: the CTNeoBC pooled analysis. Lancet 2014; 384: 164-172. https://doi. org/10.1016/S0140-6736(13)62422-8

[14] UNTCH M, JACKISCH C, SCHNEEWEISS A, CONRAD B, AKTAS B et al. Nab-paclitaxel versus solvent-based paclitaxel in neoadjuvant chemotherapy for early breast cancer (GeparSepto-GBG 69): a randomised, phase 3 trial. Lancet Oncol 2016; 17: 345-356. https://doi.org/10.1016/S14702045(15)00542-2

[15] ZHU T, LIU CL, ZHANG YF, LIU YH, XU FP et al. A phase II trial of dose-dense (biweekly) paclitaxel plus carboplatin as neoadjuvant chemotherapy for operable breast cancer. Breast Cancer Res Treat 2016; 156: 117-124. https://doi. org/10.1007/s10549-016-3735-x

[16] FOUNTZILAS G, KALOFONOS HP, DAFNI U, PAPADIMITRIOU C, BAFALOUKOS D et al. Paclitaxel and epirubicin versus paclitaxel and carboplatin as first-line chemotherapy in patients with advanced breast cancer: a phase III study conducted by the Hellenic Cooperative Oncology Group. Ann Oncol 2004; 15: 1517-1526. https://doi. org/10.1093/annonc/mdh395

[17] VON MINCKWITZ G, SCHNEEWEISS A, LOIBL S, SALAT C, DENKERT C et al. Neoadjuvant carboplatin in patients with triple-negative and HER2-positive early breast cancer (GeparSixto; GBG 66): a randomised phase 2 trial. Lancet Oncol 2014; 15: 747-756. https://doi.org/10.1016/ S1470-2045(14)70160-3 
[18] COUDERT BP, LARGILLIER R, ARNOULD L, CHOLLET P, CAMPONE $M$ et al. Multicenter phase II trial of neoadjuvant therapy with trastuzumab, docetaxel, and carboplatin for human epidermal growth factor receptor2 -overexpressing stage II or III breast cancer: results of the GETN(A)-1 trial. J Clin Oncol 2007; 25: 2678-2684. https:// doi.org/10.1200/JCO.2006.09.9994

[19] BASCH E, IASONOS A, MCDONOUGH T, BARZ A, CULKIN A et al. Patient versus clinician symptom reporting using the National Cancer Institute Common Terminology Criteria for Adverse Events: results of a questionnairebased study. Lancet Oncol 2006; 7: 903-909. https://doi. org/10.1016/S1470-2045(06)70910-X
[20] EJLERTSEN B, TUXEN MK, JAKOBSEN EH, JENSEN $\mathrm{MB}, \mathrm{KNOOP}$ AS et al. Adjuvant Cyclophosphamide and Docetaxel With or Without Epirubicin for Early TOP2ANormal Breast Cancer: DBCG 07-READ, an Open-Label, Phase III, Randomized Trial. J Clin Oncol 2017; 35: 26392646. https://doi.org/10.1200/JCO.2017.72.3494

[21] ECKHOFF L, KNOOP AS, JENSEN MB, EJLERTSEN B, EWERTZ M. Risk of docetaxel-induced peripheral neuropathy among 1,725 Danish patients with early stage breast cancer. Breast Cancer Res Treat 2013; 142: 109-118. https://doi. org/10.1007/s10549-013-2728-2

[22] BAO T, SEIDMAN AD, PIULSON L, VERTOSICK E, CHEN X et al. A phase IIA trial of acupuncture to reduce chemotherapy-induced peripheral neuropathy severity during neoadjuvant or adjuvant weekly paclitaxel chemotherapy in breast cancer patients. Eur J Cancer 2018; 101: 12-19. https://doi.org/10.1016/j.ejca.2018.06.008 